\title{
On the Modeling and Control of an Experimental Harvester Machine Manipulator
}

\author{
Evangelos Papadopoulos ${ }^{1}$, Real Frenette ${ }^{2}$, Bin $\mathrm{Mu}^{1}$, and Yves Gonthier ${ }^{1}$ \\ ${ }^{1}$ Department of Mechanical Engineering \& Centre for Intelligent Machines \\ McGill University, Montreal, PQ, Canada H3A 2A7 \\ ${ }^{2}$ Centre de Recherche Informatique de Montréal (CRIM), Montreal, PQ, Canada H3A 2N4
}

\begin{abstract}
This paper focuses on the modeling, parameter estimation, and model validation in open and closed-loop of an experimental forestry machine manipulator. Symbolic Newton-Euler and linear graph methodologies are used in deriving mathematical models of the swing, boom and stick subsystems. Actuation dynamics are integrated with manipulator dynamics to result in a complete manipulator and actuation model. Identification procedures employed in estimating physical parameters are discussed. Model validation studies show good agreement between model predictions and experiments. The models will be used for designing a controller for coordinated endpoint motion and for a real-time graphical training simulator.
\end{abstract}

\section{Introduction}

Forestry is Canada's most important industry in terms of people employed and contribution to the economy, [1]. However, increased competition from overseas and strict environmental laws require that forestry resources are harvested more efficiently and more carefully than previously. This requires sophisticated forestry equipment with better and easier to use controls, increased efficiency, and self-diagnostics. Such equipment will allow operators to concentrate at planning tree harvesting operations.

These requirements can be met by computerizing forestry machines, by including advanced control systems, enabling operators to command a machine's manipulator in Cartesian space. However, such improvements should use proven industrial grade technology, so that reliability and maintainability of the machines is not adversely affected. Better use of forest resources and increased efficiency can also be achieved by improved operator training. Such training can be facilitated greatly by the use of machine graphical simulators. The work described in this paper is concerned with modeling of a complete experimental forestry machine for the purpose of control, simulator development, and diagnostics [2], [3].

Work on coordinated control of excavator-type of machines has began in mid-eighties by P.D. Lawrence and his team [4], [5]. In this work, an excavator end-point is joystick-commanded in cylindrical coordinates, i.e. the endpoint moves in a coordinated way on a plane, while a third degree-of-freedom is added by rotating the excavator cabin. However, an important trend in forestry machines is designing machines specifically for forestry operations. Such machines have appropriate workspace size and shape, are lightweight, maneuverable, and agile. In contrast to excavators, the operator of such a machine may be sitting in a non-rotating cabin, and commanding the manipulator in Cartesian space. In addition, machine actuation systems are being improved, and are increasingly based on fast closedcenter proportional valves, and constant pressure supplies.

While standard methods exist for modeling the dynamics of rigid body manipulators driven by electrical motors, [6], few studies describe modeling of electrohydraulic manipulator systems. Mclain et al. [7] developed dynamic models for a complete electrohydraulic actuation sub-system including a single-stage, four-way, suspension-type valve, not used in industry. With exceptions, the majority of previous work has focused on modeling of individual electrohydraulic components such as servovalves, transmission lines and actuators [8]. For example, modeling and identification of transmission lines can be found in [9], of actuators in [10], and of servovalves in [11].

In this paper, we study the dynamics of a forestry machine manipulator and its electrohydraulic actuation systems. The linear graph method is implemented in deriving mathematical models for the swing, boom and stick actuation subsystems. Actuator dynamics are integrated with manipulator dynamics to result in a complete machine model. Identification procedures employed in estimating physical parameters are discussed. Model validation studies show good agreement between the model and experiments, both in open and closed loop trials. The derived models provide valuable help in the dynamic analysis of forestry machines, as well as in closed-loop and feed-forward control synthesis, and simulator design.

\section{Harvester Machine System Modeling}

The work described here is part of a recent Canadian initiative in forestry robotics, called 'ATREF' (Application des Technologies Robotiques aux Équipements Forestiers) [2]. The project's harvester machine is equipped with an articulated manipulator which includes a hydraulic motor-actuated swing joint, and cylinder-actuated boom and stick joints, see Fig. 1. At the end-point, a Hooke-type assembly permits free swinging of the processing head in two degrees-offreedom (dofs). The actuators operate at 3,000 psi, provided by two constant pressure pumps which, in turn, are driven by a diesel engine, rated $152 \mathrm{hp}$ at 2,500 rpm. Commands, 
by a human operator sitting in the cabin are processed and sent to actuators by an on-board computer system.

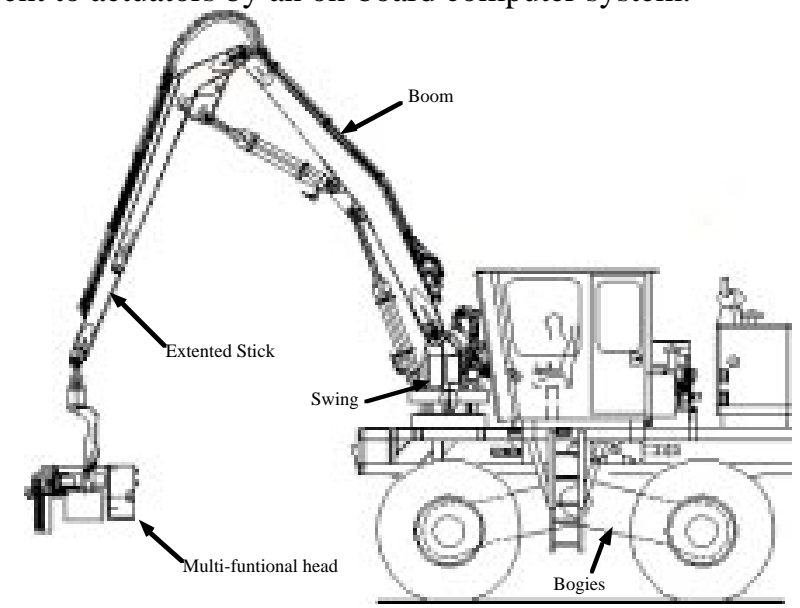

Fig. 1. The FERIC experimental machine

The harvester machine includes two main subsystems which are modeled using different techniques. The mechanical subsystem is described by the nonlinear multi-degree-offreedom rigid body dynamics of the vehicle and the manipulator and by the base compliance dynamics. The models for this subsystem are derived using a symbolic Newton-Euler approach. The electrohydraulic subsystem is described by three single-dimensional, nonlinear, and multienergy domain models, which include line, valve, actuator and power supply dynamics for the swing, boom, and stick degrees-of-freedom. This subsystem is modeled by lumped parameter elements, using the linear graph technique [12]. The two models are then integrated to form a single model in eighteen state variables, excluding the base compliance and pendulum motions of the payload. The derivation of these models is discussed below in more detail.

2a. Mechanical subsystem In contrast to industrial manipulators which are mounted on fixed bases, a forestry manipulator is mounted on a moving and compliant base introducing non-actuated dofs. These characteristics introduce additional complexity to the dynamic modelling and control of such systems.

To derive dynamic models of the machine shown in Fig. 1, the iterative Newton-Euler dynamic formulation was chosen because it is easy to implement in the form of computer code, and requires a small number of computations [6], [13]. However, this method was developed for fixedbase systems in which all dofs are actuated. In such a case, known desired trajectories for all joints, or dofs, are used to calculate numerically the forces and torques necessary to cause the desired motion. This is not possible in the case of a manipulator mounted on a compliant base, since the base is not actuated, and its position, velocity and acceleration will depend on how fast the arm moves, on the load being manipulated, etc. To avoid this problem, it was decided to apply the formulation symbolically, so as to obtain a closed set of symbolic equations of motion. The frame assignment for the derivations is shown in Fig. 2. The approach is explained in further detail in [13].

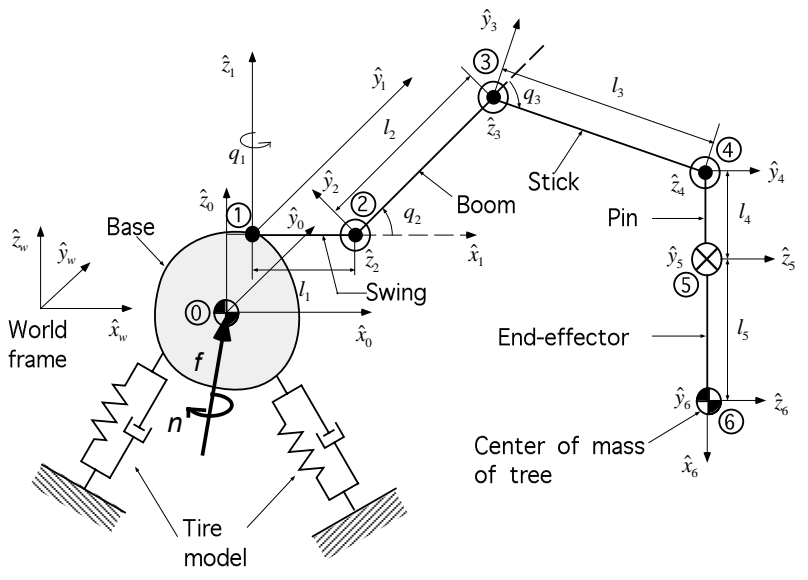

Fig. 2. FERIC machine frame assignment.

For the purposes of this work, a simpler three-dof model for the swing, boom, and stick joints was developed. The derived manipulator equations are given by

$$
\mathbf{M}(\mathbf{q}) \cdot \ddot{\mathbf{q}}+\mathbf{V}(\mathbf{q}, \dot{\mathbf{q}})+\mathbf{G}(\mathbf{q})=\boldsymbol{\tau}=\left[\tau_{s w}, \tau_{b m}, \tau_{s k}\right]^{T}
$$

where $\mathbf{q}=\left[q_{s w}, q_{b m}, q_{s k}\right]^{T}$ is the vector of joint angles, $\mathbf{M}(\mathbf{q})$ is a $3 \times 3$ mass matrix, $\mathbf{V}(\mathbf{q}, \dot{\mathbf{q}})$ includes Coriolis and centrifugal terms, $\mathbf{G}(\mathbf{q})$ includes gravity terms, $\tau_{s w}$ is the torque provided by the hydraulic motor's gearbox, and $\tau_{b m}$ and $\tau_{s k}$ are the torques on the boom and stick generated by the hydraulic cylinders.

2b. Electrohydraulic subsystem. The dynamic characteristics of hydraulic systems are intricate due to the large number of components involved and their nonlinear behavior. In this work, we have decided to use physical modelling techniques, as opposed to 'black-box' techniques. The components modeled include pumps, proportional valves, hoses, boom and stick cylinders and the swing motor. The simplest possible lumped parameter models for each component were used to simplify system identification and keep the derived models as compact as possible (a requirement for control and graphical simulator design [3]).

The boom and stick subsystems are identical in structure, and include a constant pressure power supply, modeled as a source of pressure, transmission lines, modeled as an inertance, a resistance and a capacitance connected in a $\mathrm{T}$ configuration, a valve, modeled as a nonlinear resistance modulated by an input voltage or current to the valve torque motor, and a cylinder, modeled by a double gyrator with associated damping. The electrohydraulic actuation system for the swing includes a hydraulic motor instead of a cylinder, and therefore it also includes motor internal and external leakages. More details are provided next.

The manipulator valves, selected using an inverse dynamics procedure [13], are of the two-stage, four-way proportional spool type, whose natural frequency is orders of magnitude higher than that of the desired closed-loop bandwidth. Therefore, only their resistive effect was taken into account. The valve resistance, is described by [14]

$$
\Delta P=C_{R} \cdot Q \cdot|Q|
$$

where the coefficient $C_{R}$ is a function of fluid density $\rho$, the orifice area $A$, and the discharge coefficient $C_{d}$, given as 


$$
C_{R}=\frac{\rho}{2 \cdot C_{d}^{2} \cdot A^{2}}
$$

Input voltage commands modulate the orifice area $A$, which in turn, affect the magnitude of $C_{R}$. The $C_{d}$ is fairly constant, $C_{d}=0.60$ to $0.65,[15]$.

A single T-element comprised of an inertance, a capacitance and a resistance was adequate in modelling all system hoses or transmission lines. This fact is in accordance to theory. A single element (lump) is adequate if [16]

$$
f<\frac{C_{0}}{2 \pi l}, \text { with } C_{0}=\sqrt{\frac{\beta}{\rho}}
$$

where $f$ is the line fundamental frequency of oscillation, $l$ is its length, $C_{o}$ is the velocity of sound in the fluid, and $\beta$ and $\rho$ are is its bulk modulus and density respectively. For the fluid used $\beta=1.6 \times 10^{9} \mathrm{~N} / \mathrm{m}^{2}, \rho=970 \mathrm{~kg} / \mathrm{m}^{3}$, and for the longest hose on the test vehicle of 4 meters, the wave propagation frequency $f$ is $51 \mathrm{~Hz}$, which is far above the frequencies possibly occurring in the actuation system. Since the resistance of the hoses and the valves is much higher than the resistance of filters and check valves, the effect of the latter was neglected.

The fixed-displacement, piston-type swing motor is modeled as a standard gyrator including a gearbox transformer, leakages, and viscous friction. Based on the above, the linear graph of the swing subsystem was constructed as shown in Fig. 3. In this figure, $P_{s}$ is the pump pressure; $C_{R}, C_{R^{\prime}}$ the valve orifice resistance modulated by the input current; $I_{1}, I_{2}, C_{1}, C_{2}$ and $R_{1}, R_{2}$, are the supply and return line inertance, capacitance and resistance, $R_{i n}, R_{e 1}, R_{e 2}$ the internal and external leakage of the motor whose volumetric displacement is $D_{m}, N$ is the gear train gear ratio, $B_{s w}$ is the gear train viscous damping, and $\dot{q}_{s w}$ the swing angular velocity.

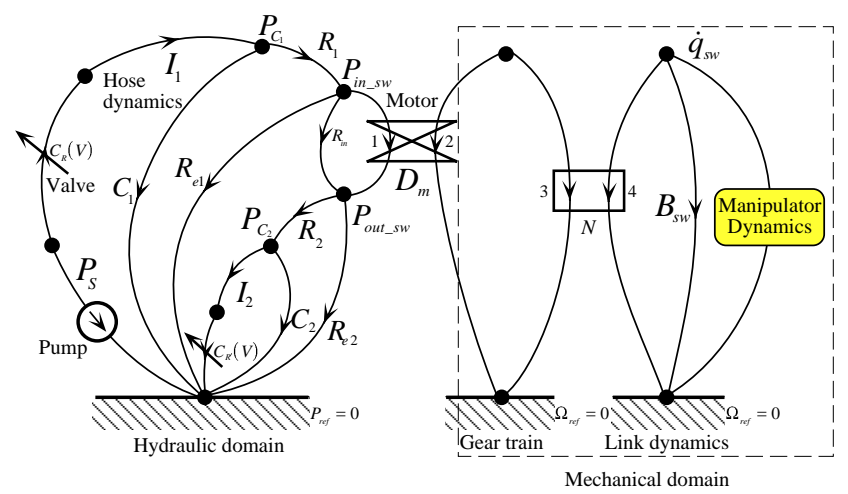

Fig. 3. The swing subsystem model.

Two single-ended type of cylinders are used to actuate the boom and stick. It is assumed that no piston leakage occurs, and that the dominant friction effects in the piston seals are viscous. The boom and stick models are constructed according to the linear graph shown in Fig. 4. Due to the single-ended configuration, the common twoport element gyrator can not be used directly. Instead, two two-port gyrators were used. Additional parameters in Fig. 4 include, the head and rod areas of the piston, $g y_{1}=A_{\text {in }}$ and $g y_{2}=A_{\text {out }}$, and the viscous cylinder damping $B$.

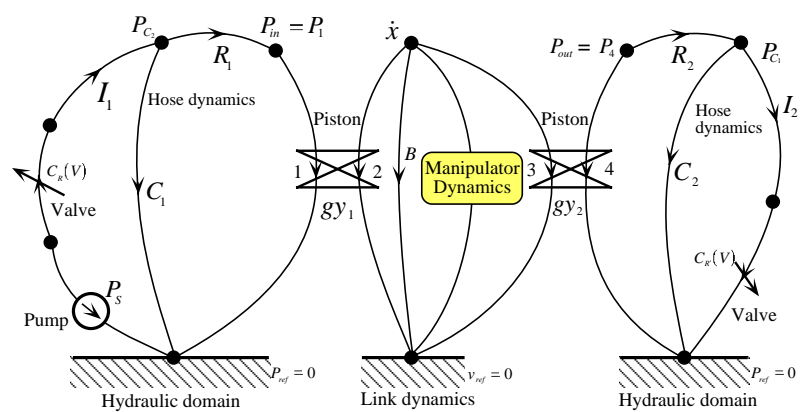

Fig. 3. Boom and stick linear graphs.

To integrate the rigid body dynamics to the electrohydraulic actuation dynamics, transduction equations transforming pressure differences to torques, and joint speeds to flows are needed. For the two single-ended cylinders, these are

$$
\begin{aligned}
& {\left[\begin{array}{c}
\tau_{b m} \\
\tau_{s k}
\end{array}\right]=\mathbf{J}^{T}\left[\begin{array}{cccc}
A_{\text {in_bm }} & -A_{\text {out_bm }} & 0 & 0 \\
0 & 0 & A_{\text {in_sk }}-A_{\text {out_sk }}
\end{array}\right]\left[\begin{array}{c}
P_{\text {in_bm }} \\
P_{\text {out_bm }} \\
P_{\text {in_sk }} \\
P_{\text {out_sk }}
\end{array}\right]} \\
& {\left[\begin{array}{c}
\dot{q}_{b m} \\
\dot{q}_{s k}
\end{array}\right]=-\mathbf{J}^{-1}\left[\begin{array}{cc}
1 / A_{\text {out_bm }} & 0 \\
0 & 1 / A_{\text {out_sk }}
\end{array}\right]\left[\begin{array}{c}
Q_{\text {out_bm }} \\
Q_{\text {out_sk }_{\text {_ }}}
\end{array}\right]}
\end{aligned}
$$

where $\mathbf{J}=\mathbf{J}(\mathbf{q})$ is a geometric Jacobian transforming joint rates to piston speeds, $A_{\text {in_bm }}, A_{\text {out_bm }}, A_{\text {in_sk }}, A_{\text {out_sk }}$ are driving and returning areas of the boom and stick pistons, $P_{\text {in_bm }}, P_{\text {out_bm }}, P_{\text {in_sk }}, P_{\text {out } s k}$ are pressures at inlet and outlet of the boom and stick cylinders, and $Q_{\text {in bm }}, Q_{\text {out bm }}, Q_{\text {in }_{s k}}$, $Q_{\text {out_sk }}$ are flow rates. The transduction equations for the swing motor are more standard, and combining the gear train ratio $\mathrm{N}$, are written as

$$
\left[\begin{array}{l}
\dot{q}_{s w} \\
\tau_{s w}
\end{array}\right]=\left[\begin{array}{cc}
0 & 1 / D_{m} N \\
-D_{m} N & 0
\end{array}\right]\left[\begin{array}{c}
P_{\text {in_sw }_{-}}-P_{\text {out }} s w \\
Q_{s w}
\end{array}\right]
$$

Finally, the system dynamic equations for the three degree of freedom manipulator, and its actuation system are written as

$$
\begin{aligned}
& \dot{\mathbf{q}}_{1}=\mathbf{q}_{2} \\
& \dot{\mathbf{q}}_{2}=\mathbf{M}\left(\mathbf{q}_{1}\right)^{-1}\left\{-\mathbf{V}\left(\mathbf{q}_{1}, \mathbf{q}_{2}\right)-\mathbf{G}\left(\mathbf{q}_{1}\right)+\tau\right\} \\
& \dot{P}_{c_{1-} s w}=\left(Q_{i_{1} s w}-\left(P_{C_{1-} s w}-P_{R_{i n}}\left(\dot{q}_{s w}\right)-P_{R_{2}}-P_{C_{2}-s w}\right) / R_{1}\right) / C_{1_{-} s w} \\
& \dot{P}_{c_{2}-s w}=\left\{\left(P_{C_{1}-s w}-P_{R_{i n}}\left(\dot{q}_{s w}\right)-P_{R_{2}}-P_{C_{2 \_} s w}\right) / R_{1}-\right. \\
& \left(P_{R_{i n}}\left(\dot{q}_{s w}\right)+P_{R_{2}}+P_{C_{2-} s w}\right) / R_{e 1} \\
& \left.-\left(P_{C_{2} \_s w}+P_{R_{2}}\right) / R_{e 2}-Q_{i_{2} \_s w}\right\} / C_{2_{-} s w} \\
& \dot{Q}_{i_{1} \leq s w}=\left(P_{s}-C_{R} \cdot Q_{i_{1}-s w}{ }^{2} \cdot \operatorname{sign}\left(Q_{i_{1} s w}\right)-P_{C_{1} s w}\right) / I_{1_{-} s w} \\
& \dot{Q}_{i_{2} s w}=\left(P_{C_{2} s w}-C_{R^{\prime}} \cdot Q_{i_{2_{-}} s w}{ }^{2} \cdot \operatorname{sign}\left(Q_{i_{2} s w}\right)\right) / I_{2_{-} s w} \\
& \dot{P}_{c_{1-b m}}=\left(Q_{i_{1-b m}}-g y_{1} \cdot \dot{x}_{b m}\right) / C_{1_{-} b m}
\end{aligned}
$$




$$
\begin{aligned}
& \dot{P}_{c_{2-} b m}=\left(g y_{2} \cdot \dot{x}_{b m}-Q_{i_{2} b m}\right) / C_{2_{-} b m} \\
& \dot{Q}_{i_{1} b m}=\left(P_{s}-C_{R} \cdot Q_{i_{1-} b m}{ }^{2} \cdot \operatorname{sign}\left(Q_{i_{1_{-}} b m}\right)-P_{c_{1_{-}} b m}\right) / I_{1_{-} b m} \\
& \dot{Q}_{i_{2} b m}=\left(P_{c_{2-} b m}-C_{R^{\prime}} \cdot Q_{i_{2-} b m}{ }^{2} \cdot \operatorname{sign}\left(Q_{i_{2} b m}\right)\right) / I_{2_{-} b m} \\
& \dot{P}_{c_{1-} s k}=\left(Q_{i_{1-} s k}-g y_{1} \cdot \dot{x}_{s k}\right) / C_{1_{-} s k} \\
& \dot{P}_{c_{2}-s k}=\left(g y_{2} \cdot \dot{x}_{s k}-Q_{i_{2}-s k}\right) / C_{2_{-} s k} \\
& \dot{Q}_{i_{1}-s k}=\left(P_{s}-C_{R} \cdot Q_{i_{1-} s k}{ }^{2} \cdot \operatorname{sign}\left(Q_{i_{1}-s k}\right)-P_{c_{1-} s k}\right) / I_{1_{-} s k} \\
& \dot{Q}_{i_{2} s k}=\left(P_{c_{2}-s k}-C_{R^{\prime}} \cdot Q_{i_{2_{-}} s k}{ }^{2} \cdot \operatorname{sign}\left(Q_{i_{2} s k}\right)\right) / I_{2_{-} s k}
\end{aligned}
$$

where $\mathbf{q}_{\mathbf{1}}=\mathbf{q}$ and $\tau$ have been defined in Eq. 1 .

\section{Experimental Parameter Estimation}

The enormous size and weight of the experimental system (e.g. its weight is about 20 ton), made identification experiments challenging. Mechanical parameters were estimated by direct measurements, by weighing disassembled link parts, and by pendulum experiments for determining inertia properties. However, the later are quite sensitive to period of oscillation errors. Therefore, detailed AutoCAD solid models of all the link parts were created, and inertia properties were computed numerically. These models were refined to the point that part mass and center of mass location matched the experimentally determined ones [13].

Most of the electrohydraulic system parameters were identified individually in order to reduce estimation errors to minimum. Standard identification procedures were required for the rest. Various types of sensors were used for these experiments: pressure transducers, resolvers, flow meters, etc. The data-acquisition system was based on a STD32-bus Ziatech-8902, 486 DX-2 computer installed in the vehicle's cabin. This embedded system runs under QNX real-time operating system, and the data sampling rate can reach $200 \mathrm{~Hz}$. The data was collected and sent to a remote 486 DX-2 computer, also running QNX.

(a) Valves. Since the three valves used for the swing, boom and stick are identical, only one of them was tested and its $C_{R}$ measured. By varying the magnitude of input voltage commands, several sets of pressures $P_{\text {in }}, P_{\text {out }}$ and flow rates $Q$ were collected. Using Eq. (1) and a MATLAB curve fitting algorithm, a polynomial representation of $C_{R}^{-1}(V)$ was found, shown in Fig. 5. The region between 0 and 1.2 volt corresponds to the deadband of the valve.

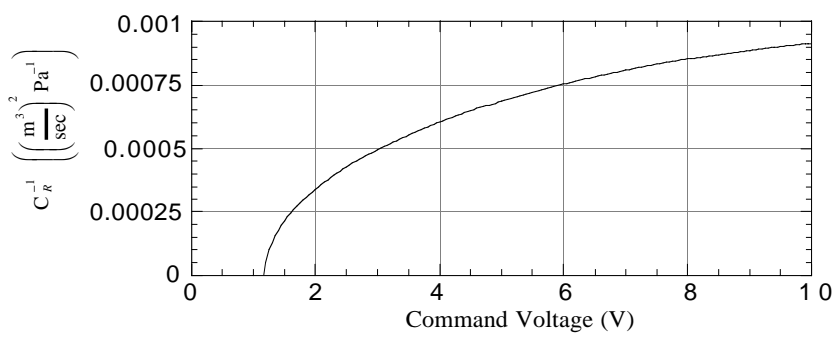

Fig. 5. Valve characteristics. (b) Hose resistance. For incompressible, fully developed turbulent flow in hoses, pressure drop is related to flow according to Eq. (1). By varying valve orifice, flow rates and corresponding pressure differences across a SAE 100 R12 hose of 4 meters in length and 3/4" in diameter were measured, and the $C_{R}$ was computed to be $3.125 \mathrm{e}-11$ $\mathrm{Pa} /\left(\mathrm{m}^{3} / \mathrm{sec}\right)^{2}$. For hoses of different diameter and length, their resistance can be approximated using

$$
C_{R}(L, D)=a \frac{\mu^{0.25} \rho^{0.75} L}{D^{4.75}}
$$

where $\alpha$ is a constant depending on the units, $\mu$ is the absolute viscosity, $\rho$ is the fluid density, $L$ is the pipe length, and $D$ is the internal diameter of the pipe, [17].

(c) Inertance and capacitance. The fluid inertance and capacitance were estimated from the pressure and flow rate readings according to their definitions. The same hose as in the hose resistance experiment was used, and flows and pressures at both ends of the hose were measured as the valve orifice was being varied. The obtained results were $\mathrm{C}$ $=1.59 \times 10^{-12} \mathrm{~m}^{5} / \mathrm{N}$, and $\mathrm{I}=3.06 \times 10^{7} \mathrm{~kg} / \mathrm{m}^{4}$.

(d) Motor leakage. The motor internal or cross-port leakage between higher and lower pressure chambers, and the external leakage from each motor chamber to the case drain were estimated assuming that leakage flows are proportional to pressure differences [17].

The internal leakage, $R_{\text {in }}$, is defined by

$$
\Delta P=P_{1}-P_{2}=R_{i n} \cdot Q_{i n}
$$

where $R_{i n}$ is the internal leakage resistance, $Q_{i n}$ is the leakage flow, and $\Delta P$ is the pressure difference across the motor ports. To estimate $R_{\text {in }}$, the platform was tilted causing the swing to rotate under gravity: $\mathrm{P}_{1}$ and $\mathrm{P}_{2}$ were measured, and $\mathrm{Q}_{\text {in }}$ was calculated as $Q_{i n}=D_{m} \dot{q}_{s w} / N$. The obtained result was $R_{\text {in }}=810^{11} \mathrm{Ns} / \mathrm{m}^{5}$.

The external leakage in each piston chamber is proportional to the chamber pressure and can be written as:

$$
P_{1}=R_{e x} \cdot Q_{e x 1}, \quad P_{2}=R_{e x} \cdot Q_{e x 2}
$$

where $R_{e x}$ is the external resistance, $P_{1}$ is the pressure in forward chamber and $P_{2}$ is pressure in return chamber. To estimate $R_{e x}$, the motor was rotated and $P_{1}$ and $P_{2}$ were measured; also, $\mathrm{Q}_{\mathrm{ex}}$ was estimated by collecting the oil from the case drain during the motion. The obtained result was $R_{i n}=2.210^{12} \mathrm{Ns} / \mathrm{m}^{5}$.

(d) Damping coefficients in cylinders and motor. The physical connections of the manipulator prevented the actuators from being disconnected from the arm and base. Since it was very difficult to estimate the viscous damping $B$ without considering the effect of other parameters, viscous damping parameters were estimated after all other parameters were identified, and accumulative errors were lumped into them [17]. The obtained results were $B_{s w}=2.45 \cdot 10^{4} \mathrm{Nms} / \mathrm{rad}$ and $B_{s k}=B_{b m}=1.03 \cdot 10^{5} \mathrm{Ns} / \mathrm{m}$.

\section{Model Validation}

Open-loop validation. The model given by Eqs. 6 was implemented in Simulink. To validate it, various input voltage commands were fed to the valves, and simulated response was compared to experimentally obtained ones. Fig. 6 displays the response of key swing subsystem variables, 
for a triangular input command. The solid line stands for actual measurements and the dotted line is the prediction using the derived dynamic models by simulation.
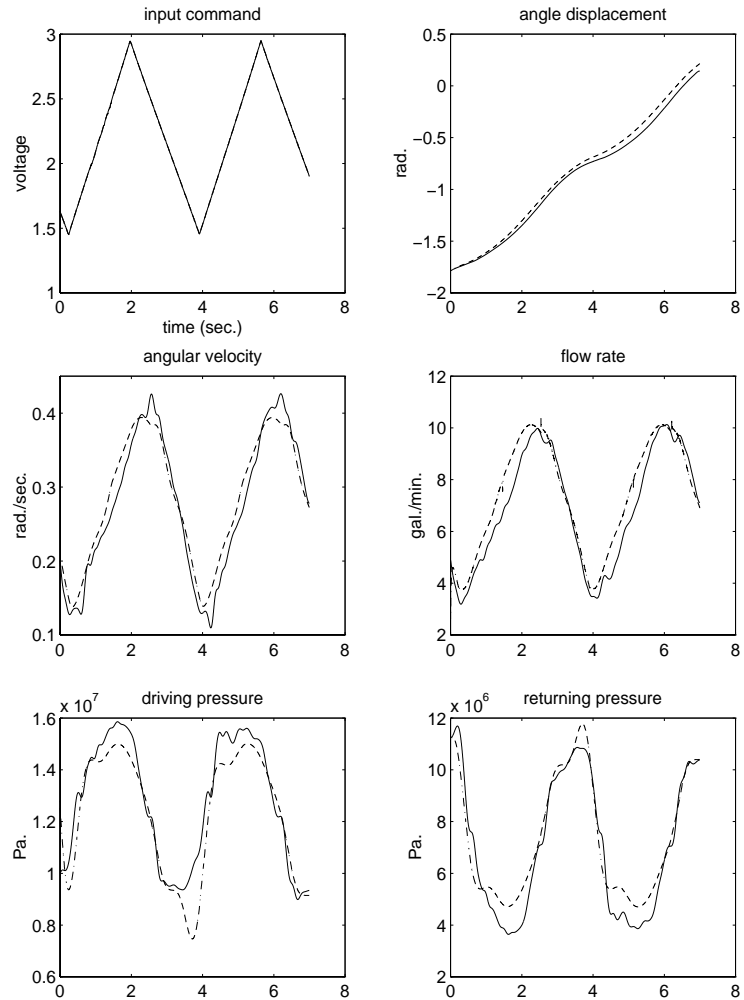

Fig. 6. Model validation studies for the swing.

The prediction of angular displacements and speeds is very close to the actual ones. The flow rate prediction is also very good because of its relation to angular rates. The predicted pressure profiles are close to the real ones, although part of the peaks was underestimated. This may be due to small discrepancies between the actual and the calculated mass properties. Furthermore, just before the experiments were conducted, a 2-DOF Hooke-type pendulum attachment was added to the stick endpoint. Periodic motions of the manipulator caused swinging motions of this attachment. Although its mass properties were known, the dynamic effects of this motion were neglected due to the lack of sensors. A more accurate friction model might also contribute in improving the results to some extent. Results for the other dofs are similar to those presented here.

Closed-loop validation. A PID controller was implemented at the joint level, see Fig. 7, without the feedforward part. This controller is part of the coordinated control scheme under development, and will be receiving joint commands computed by resolving cartesian commands into joint ones. Closed-loop validation for the stick subsystem is displayed here. A sinusoidal angle command was fed to the stick's controller. After PID gain tuning, the stick responded as shown in Figs. 8, 9 and 10. The same figures also display the response of the controlled system, simulated in Simulink, using the same PID gains. Note that the simulated and actual tracking errors are almost identical. The small oscillations close to 6 and $16 \mathrm{~s}$ are due to hose dynamics. A simplified model of the actuation system excluding these dynamics predicts accurately the mean response but it is faster, and may therefore be preferred for control or simulation purposes. Pressure predictions are good in the mean. Discrepancies are mostly due to load swinging which was not accounted for in the model.

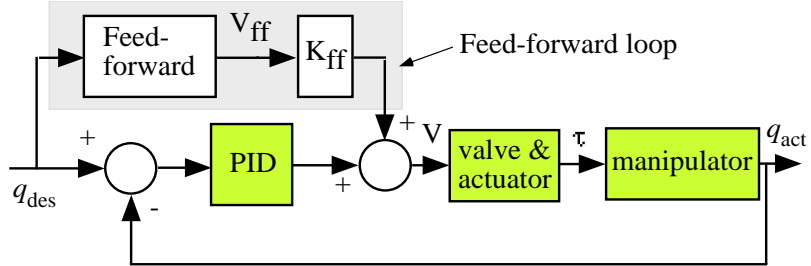

Fig. 7: PID controller.

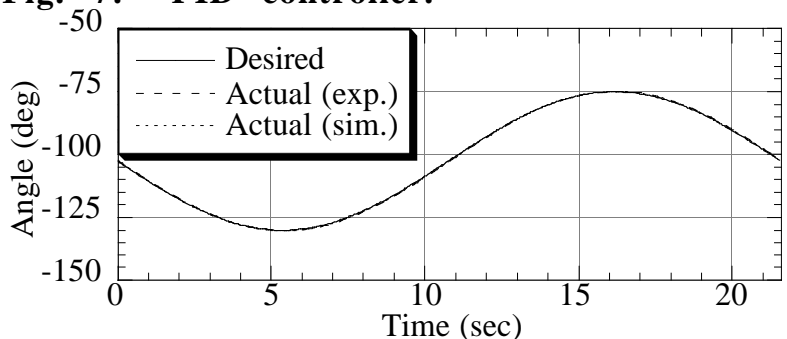

Fig. 8: Desired \& actual stick angle response.

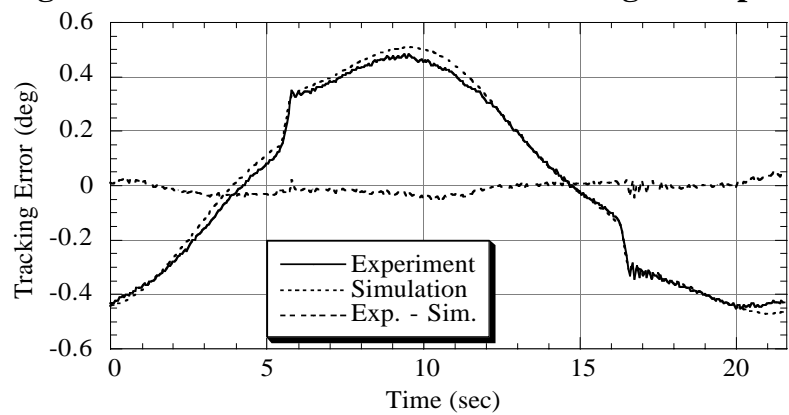

Fig. 9. Comparison of stick tracking errors

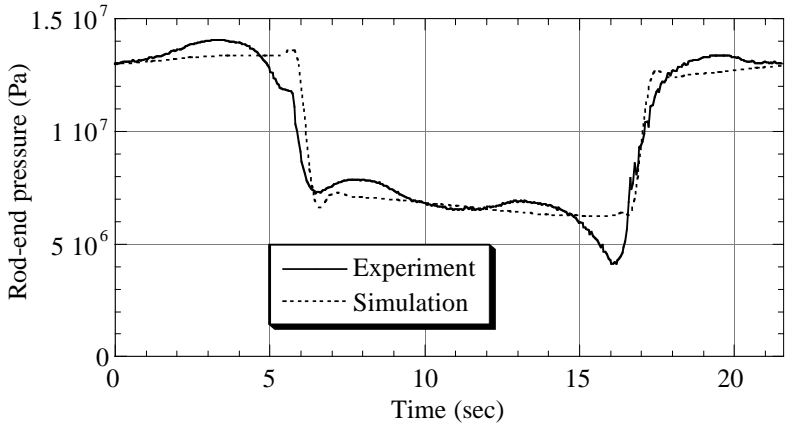

Fig. 10: Comparison of stick rod-end pressures.

\section{Feed-forward Control based on Valve Characteristics}

Tracking of the joint-level PID controller can be enhanced by adding a feedforward loop, see Fig. 7. This loop can be based on the static valve characteristic, shown in Fig. 5. Note that each of the two valve orifices are governed by Eq. (1). For the purpose of the controller, the flow $\mathrm{Q}$ for each orifice can be derived from the desired joint speed. The 
pressure drop for each orifice $\Delta P$ is obtained from four pressure transducers yielding $\mathrm{P}_{\text {pump }}, \mathrm{P}_{\mathrm{in}}, \mathrm{P}_{\text {out }}, \mathrm{P}_{\text {tank }}$. Then the corresponding $C_{R_{i}}^{-2}$ for each orifice is given by

$$
\begin{gathered}
C_{R_{1}}^{-2}=Q_{1} / \sqrt{\Delta P_{1}}=Q_{\text {des_in }} / \sqrt{P_{\text {pump }}-P_{\text {in }}} \\
C_{R_{2}}^{-2}=Q_{2} / \sqrt{\Delta P_{2}}=Q_{\text {des_out }} / \sqrt{P_{\text {out }}-P_{\text {tank }}}
\end{gathered}
$$

Using the valve characteristic depicted in Fig. 5, the voltages $\mathrm{V}_{\mathrm{ff} 1}$ and $\mathrm{V}_{\mathrm{ff2}}$ can be calculated. It was found later that using the minimum of the two gave best results.

The usefulness of the feed-forward controller has been demonstrated first off-line. The values recorded during the tests for closed-loop validation (trajectory shown in Fig. 8) were fed into a Matlab program that computes the feedforward voltages as explained above. The results are shown in Fig. 11; a difference between the feedforward and PID commands is only noticeable when the spool is moving from one side of the valve to the other (i.e. when the polarity of the command changes).

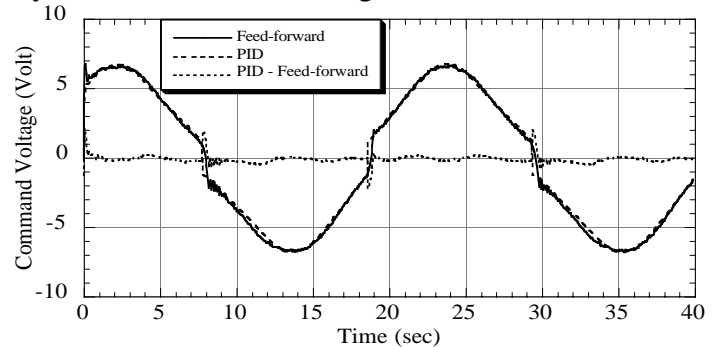

Fig. 11: Feed-forward prediction compared to PID voltage command

Since this off-line analysis revealed that the valve model alone provides a good approximation of the voltage needed to produce the desired motion, we will now proceed with incorporating the feed-forward model into our current PID controller. The gain $\mathrm{K}_{\mathrm{ff}}$ in Fig. 7 will vary between 0 and 1 and will be determined experimentally.

\section{Conclusions}

In this paper, we developed complete dynamic models for the electrohydraulic manipulator of a forestry machine. The linear graph method was implemented in deriving mathematical models for the swing, boom and stick subsystems. The actuation dynamics were integrated with manipulator dynamics to result in a complete machine model. Estimation procedures employed in obtaining values of physical parameters were discussed. Model validation studies showed good agreement between the model and experiments. The derived models are being used for feedforward and closed-loop control, and training simulator purposes. Machine behavior diagnostics will be considered also.

\section{Acknowledgments}

The financial support for this work by the Ministère de l'Industrie, du Commerce, de la Science et de la Technologie of Quebec, (MICST) under the program SYNERGIE, is gratefully acknowledged.

\section{References}

[1] Courteau, J., "Robotics in Canadian Forestry," IEEE Canadian Review, Winter 1994, pp. 10-13.

[2] Freedman, P., Papadopoulos, E., Poussart, D., Gosselin, C., and Courteau, J, "ATREF: Application des Technologies Robotiques aux Équipements Forestiers," Proc. 1995 Canadian Conf. on Electrical and Computer Eng., Montreal, PQ, Sept. 5-8, 1995.

[3] Freedman, P. MacKenzie, and J.-F. Lapointe, "A Computer-Based Training Environment for Forestry Telemanipulation," Proc. of the 1997 International Conference on Intelligent Robots and Systems (IROS '97), September 8-12, Grenoble, France.

[4] Lawrence, P.D. et al, "Computer-Assisted Control of Excavator-Based Machines," SAE Technical Paper \# 932486, Warrendale, PA, 1993.

[5] Sepehri, N. et al., "Cascade Control of Hydraulically Actuated Manipulators," Robotica, v.8, 1990, pp. 207-216.

[6] Luh, J. Y. S., Walker, M., and Paul, R. P., "OnLine Computational Scheme for Mechanical Manipulators," J. of Dynamic Systems, Measu-rement and Control, Vol. 102, 1980, pp. 69-76.

[7] McLain, T. W., et al., "Development, Simulation, and Validation of a Highly Nonlinear Hydraulic Servosystem Model," American Control Conf. Pittsburgh, Pennsylvania, June 1989, pp. 385-391.

[8] Zhou, J. J., Conrad, F., "Identification for Modeling and Adaptive Control of Hydraulic Robot Manipulators," Identification \& System Parameter Estimation 1991, No. 2, 1992, pp. 705-710.

[9] Yang, W. C., Tobler, W. E., "Dissipative Modal Approximation of Fluid Transmission lines Using Linear Friction Model," Dynamic Systems, Measurement, \& Control, Vol. 113, 1991, pp. 152-161.

[10] Wells, D. L., et al., "An Investigation of Hydraulic Actuator Performance Trade-Offs Using a Generic Model," Proc. IEEE Int. Conf. on Robotics and Automation, 1990, pp. 2168-2173.

[11] Martin, D. J., Burrow, C. R., "The Dynamic Characteristics of an Electrohydraulic Servovalve," $J$. Dynamic Systems, Measurement, \& Control, 1976.

[12] Rowell, D. and Wormley, D., System Dynamics, Prentice Hall, 1997.

[13] Papadopoulos, E. and Sarkar, S., "The Dynamics of an Articulated Forestry Machine and its Applications," Proc. IEEE Int. Conf. on Robotics and Automation, April 1997, Albuquerque, NM.

[14] Blackburn, J. F., et al., Fluid Power Control. Technology Press of M.I.T. and John Willey, 1960.

[15] Merritt, H. E., Hydraulic Control Systems. John Willey \& Sons, 1967.

[16] Watton, J., Fluid Power Systems, Prentice Hall, 1989.

[17] $\mathrm{Mu}, \mathrm{B} .$, "System Modeling, Identification and Coordinated Control Design for an Articulated Forestry Machine," Master's Thesis, Dept. of Mech. Eng., McGill University, Montreal, 1996. 